\title{
Microstructural and Crystallographic Features of Hydrogen- related Crack Propagation in Low Carbon Martensitic Steel
}

\author{
Akinobu SHIBATA, ${ }^{*}$ Hiroshi TAKAHASHI and Nobuhiro TSUJI \\ Department of Materials Science and Engineering, Kyoto University, Sakyo-ku, Kyoto, 606-8501 Japan. E-mail: shibata.akinobu. \\ 5x@kyoto-u.ac.jp
}

(Received on June 30, 2011; accepted on October 4, 2011)

\begin{abstract}
This study investigated the characteristics of hydrogen-related crack propagation in low carbon lath martensite steel through orientation analysis using electron backscattering diffraction. The orientation analysis revealed that the hydrogen-related fracture surface consisted of the $\{011\}_{M}$ facets, which were also parallel to the block boundaries or lath boundaries in the lath martensite structure. In addition, micro-cracks were observed on or in the vicinity of the prior austenite grain boundaries. On the basis of the experimental results, we proposed that hydrogen enhanced local plastic deformation occurred in the vicinity of prior austenite grain boundaries. The mechanism of hydrogen-related fracture is characterized by the formation of micro-cracks around prior austenite grain boundaries and subsequent crack propagation along block boundaries or lath boundaries.
\end{abstract}

KEY WORDS: hydrogen embrittlement; low carbon steel; mechanical properties; EBSD analysis; lath martensite; packet; block; grain boundary.

\section{Introduction}

The lath martensite structure is a typical microstructure in high strength steels. Because of the recent demands for high strength steels, the importance of lath martensite structure showing high strength has been increasing. However, one of the problems for applying high strength martensitic steels to practical applications is hydrogen-related fracture, i.e., hydrogen embrittlement or delayed fracture. It is well known that the susceptibility of martensite to hydrogen embrittlement is enhanced significantly with an increase in strength. Because the introduction of hydrogen into steels during fabrication processes or from the environment is inevitable, it is necessary to overcome the technical challenges of hydrogen embrittlement. In order to overcome hydrogen-related fracture through microstructure control, many attempts have been made to reveal the relationship between microstructure and hydrogen-related fracture. Most of such previous studies have investigated hydrogen trapping behavior, ${ }^{1-7)}$ critical hydrogen content for embrittlement, ${ }^{8-10)}$ and the relationship between microstructural change in tempering and susceptibility to hydrogen embrittlement. ${ }^{11-13)}$ In addition to such factors, it is important to characterize hydrogen-related crack propagation from a microstructural view-point in lath martensite. This has not yet, however, been accomplished in detail.

It is well known that lath martensite satisfies Kurdjumov - Sachs (K-S) orientation relationship with respect to austenite, given by $\{111\}_{\mathrm{A}} / /\{011\}_{\mathrm{M}},<101>_{\mathrm{A}} / /<111>_{\mathrm{M}}$, where the subscripts $\mathrm{A}$ and $\mathrm{M}$ denote austenite and martensite, respectively. In the K-S orientation relationship, there are twenty-four equivalent crystallographic variants that are transformed from a single grain of austenite. Accordingly, the transformation to lath martensite divides an austenite grain into several structural units of different length scales, i.e., lath, block, and packet. ${ }^{14,15)}$ The martensite lath is a single crystal of martensite with the thickness of about $0.2 \mu \mathrm{m}$. Misorientation between adjacent laths is about 3 degrees. ${ }^{16)}$ The block contains many laths having nearly the same orientation. The packet consists of several blocks with the identical parallel plane relationship in the K-S orientation relationship. Usually several packets appear within a single prior austenite grain. Boundaries between adjacent blocks or adjacent laths inside a packet have the identical crystallographic plane of approximately $\{011\}_{\mathrm{M} .}{ }^{16,17)}$

According to previous studies, ${ }^{12,18,19)}$ the hydrogen-related transgranular fracture observed in low carbon martensitic steels is characterized by cleavage fractures parallel to $\{011\}_{\mathrm{M}}$ or $\{112\}_{\mathrm{M}}$ glide planes. Kim et al. ${ }^{20,21)}$ discussed that the hydrogen induced cracks propagated along martensite lath boundaries because the lath boundaries are almost parallel to $\{011\}_{\mathrm{M}}$. However, no reports have directly examined the relationship between hydrogen-related cracks and typical structural units of lath martensite, such as lath, block, packet, and prior austenite grain boundary, through precise orientation analysis. In this study, the characteristics of hydrogen-related crack propagation are investigated from the view-point of microstructure of lath martensite, and its crystallographic features are considered in details.

\section{Experimental}

An Fe-0.1C (C: 0.09, Si: <0.001, Mn: 0.02, P: $<0.001$, S: 0.004, Fe: bal. (mass\%)) steel was used in the present 
study. A cast ingot was cold-rolled to about $1.5 \mathrm{~mm}$ thickness and then austenitized at $1323 \mathrm{~K}$ for $1.8 \mathrm{ks}$ in vacuum, followed by iced-brine quenching and sub-zero cooling in liquid nitrogen. The as-quenched specimen had a fully lath martensite structure. After the heat treatment, specimens were mechanically ground down to a thickness of $1 \mathrm{~mm}$ in order to remove the decarburized layers formed during austenitization.

For evaluating the hydrogen embrittlement behavior of the material, uniaxial tensile tests at a slow strain rate were carried out at room temperature in air. Tensile test specimens with a gauge length of $10 \mathrm{~mm}$, width of $5 \mathrm{~mm}$, and thickness of $1 \mathrm{~mm}$ were prepared by spark wire-cutting. Hydrogen was introduced to the tensile test specimens by electrochemical charging in a solution of $1 \mathrm{~N} \mathrm{H}_{2} \mathrm{SO}_{4}+2 \mathrm{mg}$ $\mathrm{L}^{-1} \mathrm{As}_{2} \mathrm{O}_{3}$ at a current density of $100 \mathrm{~A} \mathrm{~m}^{-2}$ for $86.4 \mathrm{ks}$. To keep the same condition of each hydrogen-charged specimen for the tensile testing, the tensile test was started $1.8 \mathrm{ks}$ after hydrogen-charging process was finished. The tensile test was carried out at a crosshead speed of $5 \times 10^{-3} \mathrm{~mm} \mathrm{~min}^{-1}$, corresponding to an initial strain rate of $8.3 \times 10^{-6} \mathrm{~s}^{-1}$, and the tensile strain was measured by an extensometer. Two tensile tests were carried out for each uncharged and hydrogencharged specimens.

After tensile tests, a Ni layer was electrodeposited on the specimen in order to preserve the fracture surface during subsequent polishing for microstructural observation. The microstructure was observed by optical microscopy and scanning electron microscopy (SEM, FEI: XL30S-FEG) equipped with an electron backscattering diffraction (EBSD) analyzer operated at $15 \mathrm{kV}$. For the optical microscopy observations, the specimens were polished mechanically and electrolytically in a solution of $900 \mathrm{~mL}$ $\mathrm{CH}_{3} \mathrm{COOH}+100 \mathrm{~mL} \mathrm{HClO}_{4}$, and then etched in a $3 \%$ nital solution. The fracture surfaces of the specimens after tensile tests were observed by SEM. EBSD orientation mapping on

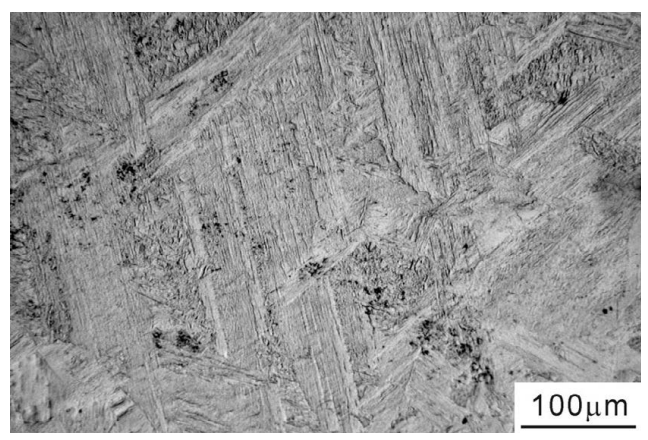

Fig. 1. Optical microscope image of the lath martensite structure in the studied steel.

Table 1. The averaged mechanical properties obtained from tensile tests of both the uncharged and hydrogen-charged specimens: $0.2 \%$ proof stress $\left(\sigma_{0.2}\right)$, ultimate tensile strength $\left(\sigma_{\mathrm{UTS}}\right)$, total elongation $\left(e_{\text {total }}\right)$, and reduction in area (RA).

\begin{tabular}{lcccc}
\hline & $\begin{array}{c}\sigma_{0.2} \\
(\mathrm{MPa})\end{array}$ & $\begin{array}{c}\sigma_{\mathrm{UTS}} \\
(\mathrm{MPa})\end{array}$ & $\begin{array}{c}e_{\text {total }} \\
(\%)\end{array}$ & $\begin{array}{c}\text { RA } \\
(\%)\end{array}$ \\
\hline uncharged specimens & 844.7 & 1068.6 & 8.31 & 36.9 \\
hydrogen-charged specimens & 870.0 & - & 2.21 & 18.0 \\
\hline
\end{tabular}

the sections parallel to the tensile direction were also carried out, in order to study the crystallographic features of hydrogen-related crack propagation. EBSD measurement and analysis were performed with the TSL OIM Data Collection program and the TSL OIM Analysis program. For SEM observations and EBSD measurements, the specimens were mechanically polished down to the thickness of $0.5 \mathrm{~mm}$, and then electrolytically polished in the same solution used for the optical microscope observation.

According to Morito et al. ${ }^{16,17)}$ or Kitahara et al., ${ }^{22)}$ martensite variants with the K-S orientation relationship can be identified by comparing the experimentally measured $\{001\}_{\mathrm{M}}$ pole figure of the specimen with the stereographic projection on which the $\langle 001\rangle_{M}$ axes of the ideal twentyfour martensite variants are plotted. This is possible because there is a certain orientation relationship between different martensite variants. After identifying the variants based on the analysis mentioned above, block boundaries with small misorientation (sometimes termed sub-block boundaries, ${ }^{16,17)}$ ) block boundaries with large misorientation, packet boundaries, and prior austenite grain boundaries in the martensite structure were determined.

\section{Results and Discussion}

Figure 1 is an optical microscope image of the asquenched specimen. The microstructure is the typical lath martensite structure where parallel aligned blocks exist inside each packet. The existence of pro-eutectoid ferrite in the specimen was not confirmed.

For the uncharged specimens and hydrogen-charged specimens, tensile tests were carried out at room temperature. The average mechanical properties obtained from the tensile tests of both uncharged and hydrogen-charged specimens, i.e., $0.2 \%$ proof stress $\left(\sigma_{0.2}\right)$, ultimate tensile strength $\left(\sigma_{\mathrm{UTS}}\right)$, total elongation $\left(e_{\text {total }}\right)$, and reduction in area (RA), are summarized in Table 1. Because the tensile test specimen is of sheet type, RA was obtained approximately from the change in the projected area along the tensile direction before and after tensile test. Figure 2 shows typical nominal stressstrain curves of the hydrogen-charged specimen (solid line) and uncharged specimen (dotted line). The uncharged specimen exhibited a large amount of post uniform elongation after reaching the tensile strength. The mechanical behavior exhibiting large post uniform elongation is typical of low

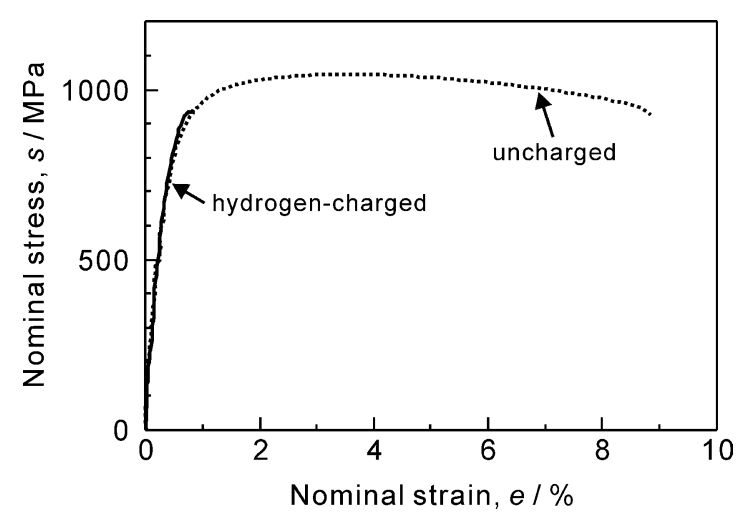

Fig. 2. Nominal stress-strain curves of the hydrogen-charged specimen (solid line) and uncharged specimen (dotted line). 
carbon martensite. ${ }^{23)}$ We find from Table 1 and Fig. 2 that the hydrogen-charged specimen exhibited limited plasticity. Compared to the uncharged specimen, the hydrogencharged specimen was fractured at a significantly lower strain.

An SEM image of the fracture surface after tensile testing of the uncharged specimen is shown in Fig. 3. The fracture surface exhibits dimple patterns typical of ductile fracture. On the other hand, two types of fracture surfaces were observed in the hydrogen-charged specimen (Fig. 4). The first is the fracture surface characterized by dimples, shown in Fig. 4(a). Compared to the uncharged specimen (Fig. 3), we find that the morphology of dimples in Fig. 4(a) are fine and shallow. The second type is the quasi-cleavage fracture surface shown in Fig. 4(b). It was reported that the quasicleavage patterns observed in hydrogen-related fracture can be regarded as dimples with quite shallow and irregular periphery. ${ }^{24)}$ Almost all areas of the fracture surface consist of dimple patterns, and quasi-cleavage fracture surfaces were not often observed. The fine and shallow dimples (Fig. 4(a)) and quasi-cleavage fracture surface (Fig. 4(b)) could be attributed to frequent void formation in uniaxial tension.

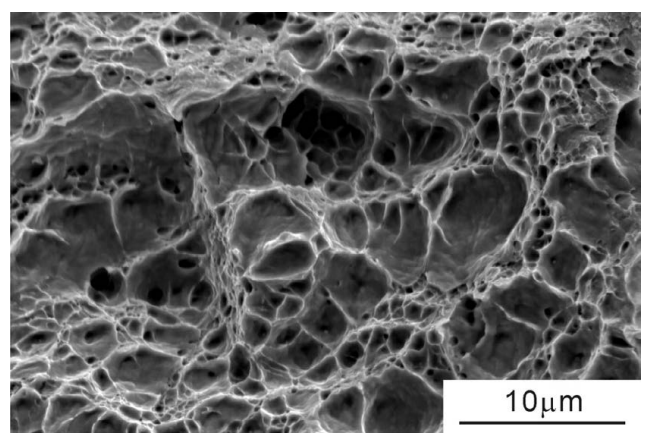

Fig. 3. SEM image showing the fracture surface of the uncharged specimen.


Fig. 4. SEM images showing two types of fracture surfaces in the hydrogen-charged specimen.
For analyzing the crystallographic features of hydrogenrelated crack propagation, orientation mapping by EBSD on the sections parallel to the tensile direction of the hydrogencharged specimen was carried out. Figure 5 is an (a) SEM image and (b) corresponding EBSD orientation map of the area in the vicinity of the fracture surface. The area of EBSD measurement (Fig. 5(b)) is indicated as a white rectangle in Fig. 5(a). The colors in Fig. 5(b) express the orientation parallel to the normal direction of the observed section, and the correspondence between color and crystal orientation is shown in the stereographic triangle inserted at right upper corner in Fig. 5(b). In addition, block boundaries with small misorientation (2-15 degree), block boundaries with large misorientation (above 15 degree), and packet boundaries are identified by gray, black, and yellow lines, respectively. Boundaries were identified through variant analysis of the lath martensite structure. ${ }^{16,17,22)}$ A prior austenite grain boundary does not exist in the area of Fig. 5(b). Because the fracture surface was preserved by the $\mathrm{Ni}$ electrodeposited layer, the exact shape of fracture surface can be observed, as shown in Figs. 5(a) and 5(b). The fracture surface of Fig. 5 corresponds to dimple fracture, because dimple patterns were observed at almost all areas of the fracture surfaces. The fracture surface consists of straight lines, indicating that the fracture mode is transgranular and the fracture surface has facets parallel to a specific set of crystallographic planes. In the EBSD orientation map, the traces of $\{011\}_{\mathrm{M}}$ planes of various martensite variants which are faced to the fracture surface are also indicated by white dotted lines. According to Fig. 5(b), it is clear that almost all facets of fracture surface are nearly parallel to the $\{011\}_{\mathrm{M}}$ plane.

Previous studies ${ }^{16,17)}$ reported that the boundaries between adjacent blocks or adjacent laths inside a packet have the identical crystallographic plane that corresponds approximately to a $\{011\}_{\mathrm{M}}$ plane. It is also noteworthy that the broad facets of fracture surface are parallel to the particular $\{011\}_{\mathrm{M}}$ plane corresponding to block boundaries or lath boundaries, although there are six crystallographically equivalent $\{011\}_{M}$ planes in a martensite crystal. This suggests that the hydrogen-related cracks propagated along block boundaries or lath boundaries inside prior austenite grain. The fracture surfaces parallel to block boundaries or lath boundaries were never observed in the uncharged specimens.

Figure 6(a) is an SEM image of an area approximately $200 \mu \mathrm{m}$ from the fracture surface in which we can observe micro-cracks. The micro-cracks are almost perpendicular to the tensile axis. Figure 6(b) is the corresponding EBSD orientation map of Fig. 6(a). Block boundaries with small misorientation (2-15 degree), block boundaries with large misorientation (above 15 degree), packet boundaries, and prior austenite grain boundaries are also identified by gray, black, yellow, and black broken lines, respectively. The position of the micro-cracks are also indicated as white lines in Fig. 6(b). We find that all observed micro-cracks are located on or in close proximity to the prior austenite grain boundary. Such a location of micro-cracks was not a special case but typical in the many observed areas. Five additional areas other than Fig. 6 were examined, and we confirmed in all five cases that the micro-cracks are located on or at close vicinity of prior austenite grain boundaries. 

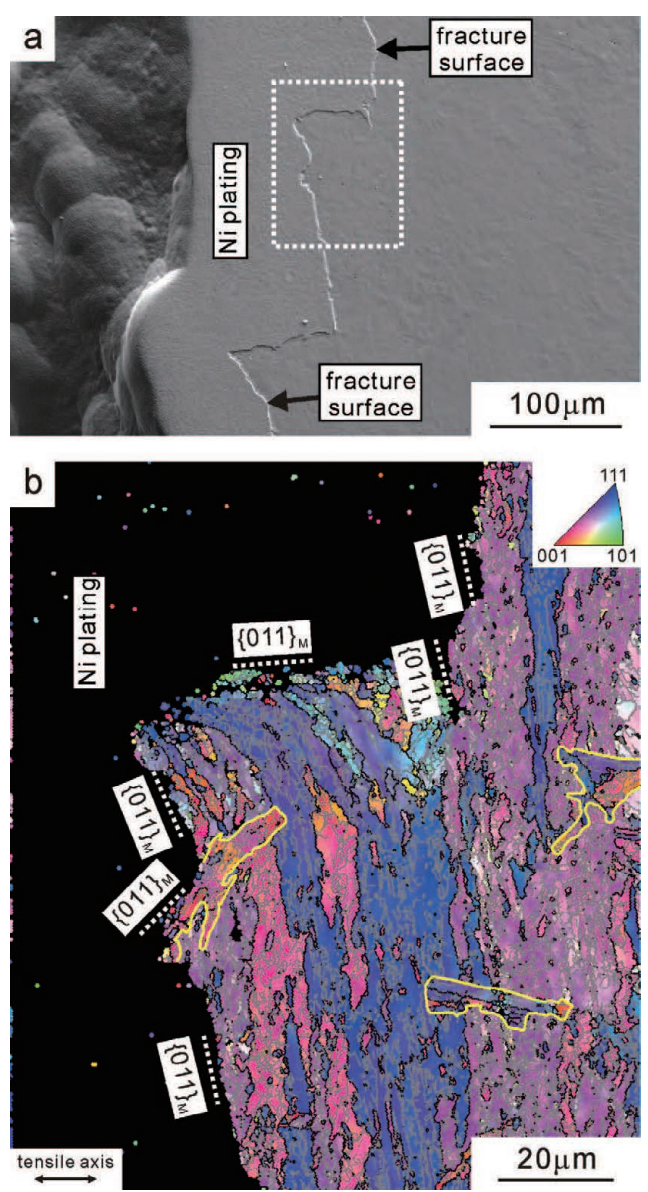

Fig. 5. (a) SEM image and (b) corresponding EBSD orientation map of an area in the vicinity of the fracture surface of the hydrogen-charged specimen after tensile test. The area of EBSD measurement shown in (b) is indicated by a white dotted rectangle in (a). Block boundaries with small misorientation (2-15 degree), block boundaries with large misorientation (above 15 degree), and packet boundaries are identified by gray, black, and yellow lines, respectively.

There have been many reports concerning the distribution of hydrogen trapping sites in steels. ${ }^{1-7)}$ Luppo and OvejeroGarcia $^{1)}$ examined the trapping site of hydrogen in lath martensite in a low carbon martensitic steel using the hydrogen microprint technique. According to reported results, the predominant trapping sites of hydrogen in lath martensite are interfaces between laths or prior austenite grain boundaries. Takai et $a l .^{2)}$ studied hydrogen trapping sites in lath martensite by using deuterium as a tracer for hydrogen. They reported, using secondary ion mass spectrometry observations, that hydrogen introduced during the delayed fracture test was trapped mainly on prior austenite grain boundaries. Hirth $^{19)}$ proposed that hydrogen enhances the mobility of dislocations based on internal friction and mechanical property studies. Moreover, Robertson et al. ${ }^{25,26)}$ directly observed the hydrogen-accelerated dislocation motion by in-situ transmission electron microscopy deformation experiments. The hydrogen-enhanced local plasticity could be attributed to the fact that hydrogen atmospheres formed at dislocations reduce the stress field of dislocations. ${ }^{26)}$ On the basis of these previous studies and experimental results, we propose that local dislocation motion in the vicinity of prior austenite grain boundaries was enhanced due to the high accumulation of hydrogen around prior austenite grain
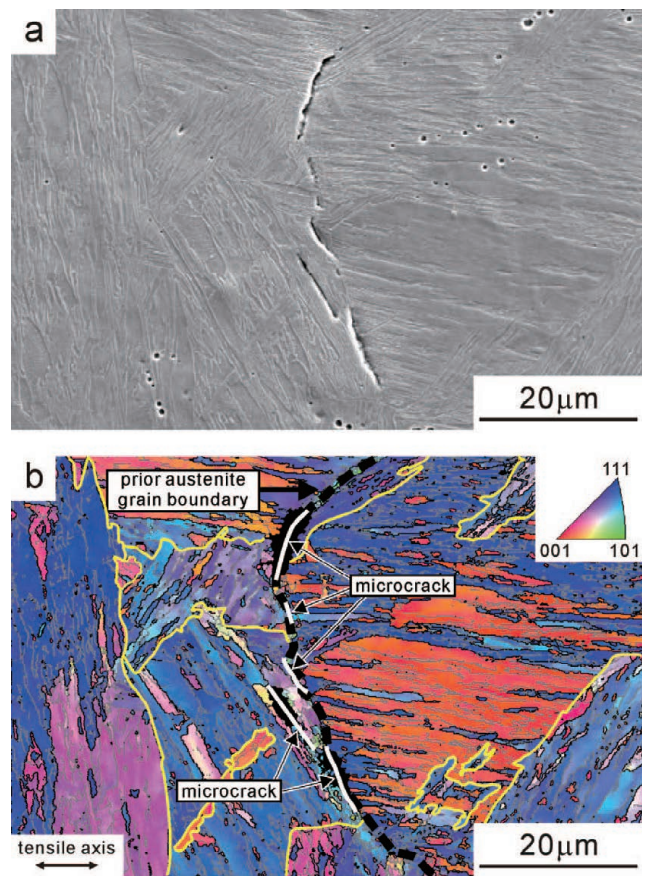

Fig. 6. (a) SEM image of an area about $200 \mu \mathrm{m}$ from the fracture surface, and (b) corresponding EBSD orientation map of the hydrogen-charged specimen after tensile test. Block boundaries with small misorientation (2-15 degree), block boundaries with large misorientation (above 15 degree), packet boundaries, and prior austenite grain boundaries are identified by gray, black, yellow, and black broken lines, respectively.

boundaries. This hydrogen-enhanced local dislocation motion resulted in the formation of micro-cracks on lath boundaries or block boundaries in the vicinity of prior austenite grain boundaries. The propagation of micro-cracks along block boundaries or lath boundaries inside prior austenite grain lead to hydrogen-related fracture.

\section{Summary}

The present paper investigated the characteristics of hydrogen-related crack propagation in a low carbon martensitic steel from both microstructural and crystallographic points of view. The major results obtained are listed as follows:

(1) Tensile tests at a slow strain rate revealed that the hydrogen-charged specimen exhibited limited plasticity, and was fractured at very small strain when compared with the uncharged specimen.

(2) The fracture surface of the hydrogen-charged specimen exhibited fine and shallow dimple patterns as well as quasi-cleavage patterns. Almost all areas of the fracture surface consisted of dimple patterns, and quasi-cleavage fracture surfaces were not often observed. This indicated that the presence of hydrogen enhanced void formation.

(3) Through orientation analysis based on EBSD measurement, we found that the fracture surface of the hydrogen-charged specimen consisted of facets parallel to $\{011\}_{\mathrm{M}}$ planes. Facets were also parallel to block boundaries or lath boundaries in the lath martensite structure. In areas away from the fracture surface, the micro-cracks were observed on or in the vicinity of a prior austenite grain boundary. The 
present results suggested that hydrogen enhanced dislocation motion in the vicinity of the prior austenite grain boundaries resulted in the formation of micro-cracks near prior austenite grain boundaries.

\section{Acknowledgement}

This study was financially supported by the Grant-in-Aid for the Global COE program, "International Center for Integrated Research and Advanced Education in Materials Science," from the Ministry of Education, Culture, Sports, Science and Technology of Japan.

\section{REFERENCES}

1) M. I. Luppo and J. Ovejero-Garcia: Corros. Sci., 32 (1991), 1125.

2) K. Takai, J. Seki and Y. Homma: Mater. Trans., JIM, 36 (1995), 1134.

3) F-G. Wei, T. Hara, T. Tsuchida and K. Tsuzaki: ISIJ Int., 43 (2003), 539.

4) M. Nagumo and H. Matsuda: Philos. Mag. A, 82 (2002), 3415.

5) F-G. Wei and K. Tsuzaki: Scr. Mater., 52 (2005), 467.

6) H. Fuchigami, H. Minami and M. Nagumo: Philos. Mag. Lett., 86 (2006), 21.

7) J. Takahashi, K. Kawakami, Y. Kobayashi and T. Tarui: Scr. Mater.,
63 (2010), 261.

8) M. Wang, E. Akiyama and K. Tsuzaki : Mater. Sci. Technol., 22 (2006), 167.

9) M. Wang, E. Akiyama and K. Tsuzaki: Corros. Sci., 49 (2007), 4081.

10) S. Li, Z. Zhang, E. Akiyama, K. Tsuzaki and B. Zhang: Corros. Sci., 52 (2010), 1660

11) B. Craig and G. Krauss: Metall. Trans. A, 11A (1980), 1799.

12) Y. Takeda and C. J. McMahon, Jr.: Metall. Trans. A, 12A (1981), 1255.

13) S-J. Lee, J. A. Ronevich, G. Krauss and D. K. Matlock: ISIJ Int., 50 (2010), 294.

14) A. R. Marder and G. Krauss: Trans. ASM, 60 (1967), 651.

15) J. M. Marder and A. R. Marder: Trans. ASM, 62 (1969), 1.

16) S. Morito, X. Huang, T. Furuhara, T. Maki and N. Hansen: Acta Mater., 54 (2006), 5323.

17) S. Morito, H. Tanaka, R. Konishi, T. Furuhara and T. Maki: Acta Mater., 51 (2003), 1789.

18) F. Nakasato and I. M. Bernsten: Metall. Trans. A, 9A (1978), 1317.

19) J. P. Hirth: Metall. Trans. A, 11A (1980), 861.

20) Y. H. Kim and J. W. Morris, Jr.: Metall. Trans. A, 14A (1983), 1883.

21) Y. H. Kim, H. J. Kim and J. W. Morris, Jr.: Metall. Trans. A, 17A (1986), 1157.

22) H. Kitahara, R. Ueji, N. Tsuji and Y. Minamino: Acta Mater., 54 (2006), 1279.

23) G. Krauss: Mater. Sci. Eng. A, 273-275 (1999), 40.

24) M. Nagumo: Tetsu-to-Hagané, 90 (2004), 766.

25) I. M. Robertson: Eng. Frac. Mech., 68 (2001), 671.

26) P. Sofronis and I. M. Robertson: Philos. Mag. A, 82 (2002), 3405. 